\title{
THE HAWC AND SAFIRE ADIABATIC DEMAGNETIZATION REFRIGERATORS
}

\author{
Jim Tuttle, Peter Shirron, Michael DiPirro, Michael Jackson, \\ Jason Behr, Evan Kunes, and Tom Hait
}

\section{Code 552, NASA/Goddard Space Flight Center}

Greenbelt, MD 2077.1

\section{ABSTRACT}

HAWC and SAFIRE are far-infrared experiments which will fly on the SOFIA aircraft. HAWC's detectors will operate at 0.2 Kelvin, while those of SAFIRE will be at 0.1 Kelvin. Each instrument will include an adiabatic demagnetization refrigerator (ADR) to cool its detector stage from the liquid helium bath temperature (HAWC's at 4.2 Kelvin and SAFIRE's pumped to about 1.3 Kelvin) to its operating temperature. Except for the magnets used to achheve the cooling and a slight difference in the heat switch design, the two ADRs are nearly identical. We describe the ADR design and present the results of performance testing.

Suggested keywords: Adiabatic Demagnetization Refrigerator, ADR, magnetic cooling

\section{INTRODUCTION}

SAFIRE (Submillimeter and Far Infrared Experiment) and HAWC (HighResolution Airborne Wide-band Camera) are far-infrared experiments which will fly on the SOFIA (Stratospheric Observatory for Infrared Astronomy) aircraft. HAWC, a facility mission, is a joint effort among the University of Chicago (UC), Goddard Space Flight Center (GSFC), and Rochester Institute of Technology. Its far-infrared camera has a spectral band of 40 to $300 \mu \mathrm{m}$, and a $12 \times 32$ array of detectors [1] operating at 0.2 Kelvin. SAFIRE is a PI instrument built by GSFC. 
Its bolometers, configured in a $6 \times 32$ array, operate at 0.1 Kelvin and are sensitive to a band of 145 to $655 \mu \mathrm{m}$.

Each of these instruments will include a cryostat with a liquid helium bath (HAWC's at 4.2 Kelvin and SAFIRE's pumped to about 1.3 Kelvin). A cooler provided by GSFC will maintain each set of detectors at its operating temperature during science operations. The coolers will be allowed one two-hour thermal cycle per mission and then will hold the detectors at temperature for 16 to 24 hours. The total heat load from the HAWC and SAFIRE detector arrays will be about $5 \mu \mathrm{W}$ and $3 \mu \mathrm{W}$ respectively. The cooler must operate autonomously, including its thermal cycle. It must be built to survive a nominal ten-year lifetime, which may include long storage periods at room temperature and a large number of thermal cycles to liquid helium temperature. In addition, the cooler must meet airworthiness certification requirements. The cooler chosen for these two projects is an adiabatic demagnetization refrigerator (ADR).

\section{ADR OVERVIEW}

ADRs are appealing for a number of reasons. They use a robust, thermodynamically efficient magnetic refrigeration cycle, and they can provide lower controlled temperatures than a pumped ${ }^{3} \mathrm{He}$ system. They are completely self-contained drop-in devices with no external plumbing and no moving parts. They are particularly well suited to maintaining a constant stage temperature, and their thermal cycle is easy to automate.

An ADR consists of a paramagnetic salt pill suspended inside a magnet bore and connected thermally to a heat sink by a heat switch. The salt's entropy depends on both temperature and magnetic field, and the heat switch and magnet allow manipulation of the system in a way that results in cooling. The salt is attached to a thermal bus which links it to the stage to be cooled. In the HAWC and SAFIRE instruments the heat sink is the helium bath, and the detector stage is cooled by the thermal bus.

The ADR cycle is illustrated in figure 1 . It begins (in step A) with zero magnetic field in the magnet bore with the salt pill at a temperature equal to or below that of the heat sink. At this point 
the heat switch is open, thermally isolating the salt pill from the bath. The magnetic field in the bore is then increased, aligning the salt's magnetic spins and causing its temperature to rise to keep the entropy constant. When the salt pill temperature reaches that of the sink, the heat switch is closed (step B). The magnetic field is increased, lowering the salt's entropy and dumping heat to the bath. When the field reaches a chosen maximum value, it is held constant while the salt pill cools to the sink temperature. The switch is then opened (step C), and the salt pill is demagnetized adiabatically. The salt temperature drops in order to maintain constant entropy. After the salt pill temperature reaches its operating point, it is controlled by demagnetizing at a rate which provides just enough cooling to balance the heat reaching it (step D). This controlling is aided by the fact that raising or lowering the magnetic field causes heating or cooling with a very short time constant. As the salt absorbs heat at constant temperature, its entropy increases. When the applied magnetic field reaches zero, the cycle is finished.

\section{DESIGN}

The HAWC and SAFIRE ADR design is based on that of the X-Ray Spectrometer (XRS) $[2,3]$ instrument on Astro-E. Heat sunk to a 1.3 Kelvin liquid helium bath, the XRS ADR was used to temperature control a 32-element array of silicon micro-calorimeters at $60 \mathrm{mK}$ with a 36-hour hold time. SAFIRE's salt mass, magnetic field, and bath temperature are the same as those of XRS, but its 0.1 Kelvin operating temperature is much higher. Thus the same entropy increase results from a much higher heat input, and SAFIRE will have a significantly longer hold time. HAWC's 16 hour hold time requirement and 0.2 Kelvin operating temperature help to compensate for the effect its higher bath temperature relative to XRS. The addition of a higher field magnet and a heat switch less thermally conductive in the open state were the only changes from the XRS design needed to meet the HAWC thermal requirements. HAWC and SAFIRE's approach of using off-the-shelf hardware where possible and relying on prior component designs eliminated developmental hardware except for the heat switch and temperature control system. 
The ADR design, not including the magnet, is shown in figure 2. The salt pill, fabricated by the University of Wisconsin's Space Science and Engineering Center, is a modified version of the XRS pill. The HAWC/SAFIRE pill contains 920 grams of ferric ammonium alum (FAA) with 800 gold wires imbedded in the salt to connect it thermally to the upper end cap. The salt pill is held in place by two suspension assemblies, shown in figure 3. Each of these assemblies includes a cold side which bolts to one end of the pill, and a warm side which attaches to the 1.3 or 4.2 Kelvin magnet mandrel. The two sides of each assembly are held relative to each other by tensioned Kevlar ropes. Based on the known thermal conductivity of Kevlar rope, the total calculated heat conducted through both assemblies is $4.3 \mu \mathrm{W}$ for the HAWC configuration and $0.2 \mu \mathrm{W}$ for SAFIRE. The upper suspension assembly, providing both lateral and axial restraint, has its warm side bolted to the top of the magnet flange. The lower assembly provides only lateral restraint. Its warm side is a small ring which slides over a pin in the bottom of the magnet bore, grabbing the pin tightly due to differential contraction on cooldown. A rigid copper bus, bolted to the pill's upper end, extends through the upper suspension assembly. A $16.5 \mathrm{~cm}$ long thermal strap made of three $0.5 \mathrm{~mm}$ thick $\times 2.54 \mathrm{~cm}$ wide copper strips links the top of this bus to the top of the heat switch. This strap includes an "S" bend to reduce its stiffness. Another strap, made of copper braid, will connect the bus to the detector assembly.

The HAWC and SAFIRE magnets were manufactured by American Magnetics. Each is shielded by a bucking coil at the front end, and the SAFIRE magnet has additional passive shielding which has been described elsewhere[4]. The HAWC magnet produces a maximum field of 8 Tesla with a 55 amp current, with an average field in its bore volume of 7.3 Tesla. The SAFIRE magnet's field is 2 Tesla at its operating current of 25 amps.

\section{Heat Switch Design}

The heat switch, shown in detail in figure 4 , is a ${ }^{3} \mathrm{He}$ gas-gap variety similar to that used on XRS [5]. It consists of two concentric copper conductors inside a composite tube. Each conductor includes a flange which seals one end of the tube. The bottom conductor flange, or switch base, is bolted to the magnet (at the helium bath temperature). The top conductor flange is bolted to the 
thermal strap which links it to the salt pill bus. The tube holds the two conductors parallel, maintaining a nominal $0.25 \mathrm{~cm}$ gap between them. It also contains the ${ }^{3} \mathrm{He}$ gas which thermally shorts the conductors together when the switch is closed. To open the switch, a zeolite getter plumbed into the tube volume is allowed to cool below about 15 Kelvin. The getter then adsorbs all of the ${ }^{3} \mathrm{He}$, eliminating the gaseous thermal conduction. In this open state, the parasitic heat conduction through the tube is several orders of magnitude lower than the closed switch conduction. To close the switch, power is applied to the getter via a heater bolted to its can. When the getter's temperature reaches about 17 Kelvin it releases enough ${ }^{3} \mathrm{He}$ gas to provide the switch's closed conductance.

The tube is a gamma-alumina composite with a $12.7 \mu \mathrm{m}$ thick titanium 15-3-3-3 foil bonded to its inner surface. It was developed originally as a leak-tight neck tube for the GP-B cryostat [6]. This configuration was chosen for the switch to provide very low thermal conductance, to be impervious to helium, and to survive multiple thermal cycles. It also eliminates out-gassing materials from the interior of the switch, reducing the likelihood of getter contamination effects seen in the XRS switch[5]. The permeation of ${ }^{3} \mathrm{He}$ gas through the HAWC switch tube was measured at room temperature. With a pressure drop of just over one atmosphere, the gas permeated at a rate of less than $10^{-8}$ standard cc. per second. Given the switch gas volume of $6.1 \mathrm{~cm}^{3}$, this implies a half life of more than 13 years.

In the first set of tubes made for HAWC and SAFIRE, the gamma alumina fibers were wound at a 45 degree angle to the tube axis to a wall thickness of $330 \mu \mathrm{m}$. A heat switch was made with one of tubes, but its open-state thermal conductance from 4.2 to 0.2 Kelvin was too high to meet HAWC's requirements. Its 1.3 to 0.1 Kelvin conductance met SAFIRE's needs, so it became the SAFIRE switch. New tubes were purchased having a $80-85$ degree wrap angle and $280 \mu \mathrm{m}$ wall thickness, and the HAWC switch was made using one of these. The data from these tests are presented in the performance section of this paper. Subsequently a $5.3 \mathrm{~cm}$ long $\times 5.1 \mathrm{~cm}$ wide $\mathrm{x}$ $0.16 \mathrm{~cm}$ thick sample of titanium 15-3-3-3 was procured from Supra Alloys (Camarillo, CA), and 
its thermal conductance was measured in the relevant temperature range. The resulting thermal conductivity data are shown in figure 5 .

\section{ADR Control System}

The ADR salt pill temperature is read out using ruthenium oxide thermometers. In the flight configuration the detector stage temperature will be controlled, but in testing mode the salt pill's thermal bus temperature was controlled. The thermometer, with a resistance of about $50 \mathrm{~K} \Omega$ at 0.2 Kelvin, is read out with a Linear Research LR-700 bridge. The bridge's analog output is read about seven times per second by a Keithley 2000 volt meter, to which the control system's PC talks via the GPIB. The magnet current during cycling is provided by an American Magnetics four quadrant power supply, and during temperature control at 0.2 Kelvin by a Keithley 2420 voltage source. This latter source is used in a four-wire mode with voltage feedback from the magnet leads at the magnet.

The control software is a Labview ${ }^{\mathrm{TM}}$ program developed at GSFC. It includes a PID control routine based on Labview's control tool set. The program cycles the ADR using the following steps: turn on the heat switch getter heater, wait for the getter to reach 18 Kelvin, command the large power supply to ramp the current up to 30 amps at a rate of 0.0666 amps per second, wait at maximum field for the salt pill to cool to below 5 Kelvin, power off the getter heater, wait for the getter to cool below 6 Kelvin, and ramp the current down to 2 amps. Then it transitions to the Keithley 2420 voltage source to establish temperature control at 0.2 Kelvin. In this mode the PID routine raises and lowers the current by applying a positive or negative voltage across the magnet. The resulting changes in the magnetic field produce the heating or cooling required for temperature control.

\section{PERFORMANCE}

The ADR's cooling capacity is determined largely by the salt mass, the demagnetization temperature, the magnetic field, and the operating temperature. Assuming $70 \%$ efficiency and a 4.5 Kelvin demagnetization temperature, HAWC's ADR was predicted to meet its 16 hour hold time 
requirement at 0.2 Kelvin with a total heat input of up to $53 \mu \mathrm{W}$. SAFIRE was expected to achieve a 90 hour hold time at 0.1 Kelvin with a total heat input of $2 \mu \mathrm{W}$, leaving plenty of margin on the requirement. The total input includes heat from the detector stage as well as parasitic contributions from the open-state heat switch, the Kevlar suspension system, thermometer leads, and radiation.

The ADR's recycle time is determined by the magnet's current ramping rate, the heat switch's closed-state conductance, and the time required to fully open the switch before demagnetization.

\section{Heat Switch Performance}

The open-state conductance was measured for both the HAWC and SAFIRE heat switches before they were integrated with their respective ADR assemblies. In each of these tests the switch base was mounted on a laboratory ADR's cold stage and held at the HAWC/SAFIRE ADR's operating temperature. Then heat applied to the switch's opposite end was adjusted until that end reached the appropriate bath temperature. This process took several hours to complete due to the switches' long time constants. The HAWC switch's conductance from 4.2 to 0.2 Kelvin was found to be $10.3 \mu \mathrm{W}$. The SAFIRE switch conducted $1.87 \mu \mathrm{W}$ from 1.39 to 0.2 Kelvin and 18.6 $\mu \mathrm{W}$ from 4.05 to 0.2 Kelvin.

The closed-state conductances were measured in a similar fashion. The switch base was held at a temperature, the switch was closed, and heat was applied to the opposite end to establish a small delta $T$. In one sense these measurements were easier to make, as the time constants were much shorter in the closed state. However, in this configuration the large getter heat (a few $\mathrm{mW}$ )was dumped through the switch base to the lab ADR. This shortened the lab ADR's hold time drastically, making the lower temperature points difficult to measure. The closed state conductances for the HAWC and SAFIRE switches are shown in table 1. Assuming that the two switches' geometries are identical, the data show a significant getter temperature dependence. This indicates that the mean free path of the gas in the switch is comparable to or larger than the switch gap distance. 


\section{Performance of Full Assembly}

The HAWC ADR was tested in an engineering mode which simulated the flight configuration but did not include the detector heat load. While the magnet's maximum achievable current is $50 \mathrm{amps}$, the performance test was done to $30 \mathrm{amps}$. The ADR was cycled from its initial zero field/4.2 Kelvin state to the point when temperature control was established at $0.2 \mathrm{Kelvin}$ in just less than one hour. The maximum field was 4.5 Tesla, and the hold time at this temperature was 24.7 hours. A second cycle was performed with the parasitic load and an added heat of $5 \mu \mathrm{W}$. While controlling the salt pill at its operating temperature, the demagnetization rate with just the parasitic heat load was compared to the rate with an added load of $15 \mu \mathrm{W}$. From these two rates it was possible to calculate the parasitic heat load, which was $18.5 \mu \mathrm{W}$. When multiplied by the hold time, this gives a cooling capacity of 1.30 Joules at the operating temperature of $0.2 \mathrm{Kelvin}$.

The SAFIRE ADR was tested in its approximate flight configuration: the bath temperature was 1.27 Kelvin, the maximum field was 2 Tesla. The parasitic heat load was found to be $2.0 \mu \mathrm{W}$, and the hold time was projected to be about 100 iours (it was not actually demagnetized to zero current).

\section{Temperature Control}

The HAWC/SAFIRE temperature stability requirement is: for $\mathrm{f}>1 \mathrm{~Hz}$ temperature noise spectral density $<3 \times 10^{-7} \mathrm{~K} / \sqrt{\mathrm{Hz}}$; for $3 \mathrm{mHz}<\mathrm{f}<1 \mathrm{~Hz}$ temperature fluctuations $<3 \times 10^{-6} \mathrm{~K} \mathrm{~ms}$; for $\mathrm{f}<3 \mathrm{mHz}$ temperature drift $<3 \times 10^{-5} \mathrm{~K}$. During SAFIRE's performance testing the salt pill temperature values were analyzed using an FFT routine. Figure 6 shows the noise spectrum during the temperature control phase. The horizontal lines indicate the equivalent white noise densities which would just meet the requirements in the three frequency bands. The data were not stored for long enough to study the very low frequency noise, but above about one $\mathrm{mHz}$ the drift is several orders of magnitude lower than the allowed limit. The temperature fluctuation due to the middle band frequencies was $1.7 \mu \mathrm{K} \mathrm{rms}$, meeting the requirement by almost a factor of two. Above one $\mathrm{Hz}$ the thermometry noise spectral density exceeded the limit up to about $3 \mathrm{~Hz}$. However, the 
ADR's thermal time constant is long enough to prohibit significant temperature fluctuations above about one $\mathrm{Hz}$, so the temperature stability requirement has actually been met.

\section{CONCLUSION}

By using almost identical designs on the HAWC and SAFIRE ADRs and using existing technology where possible, both projects were able to achieve significant cost savings. In addition to producing the required refrigerators for these two projects, this effort provided the opportunity to develop and test a new variation on the ${ }^{3} \mathrm{He}$ gas gap heat switch design and a new suspension system. In addition, a new temperature control rack and software were developed. The performance testing indicated that both of these ADR systems meet the requirements of their respective instruments, and they are now being stored in readiness for integration. They will fly on the SOFIA aircraft at some time after 2002.

\section{ACKNOWLEDGMENTS}

The authors are grateful to David Sohl for his help with the temperature control system and to John Bichell for his assistance with the assembly and testing. This work was funded by NASA's Office of Space Science.

\section{REFERENCES}

1 D.J. Benford, C.A. Allen, A.S. Kutyrev, S.H. Moseley, R.A. Shafer, J.A. Chervenak, E.N. Grossman, K.D. Irwin, J.M. Martinis \& C.D. Reintsema. Superconducting Bolometer Arrays for Submillimeter Astronomy, 1999, Proceedings of "Imaging at Radio through Submillimeter Wavelengths," ASP \#217 (1999) 134.

2 Serlemitsos A.T., SanSebastian M, and Kunes E. Final Design of the Astro-E/XRS ADR, Advances in Cryogenic Engineering (1998) 43957. 
3 Susan R. Breon, Michael J. DiPirro, James G. Tuttle, Peter J. Shirron, Brent A. Warner, Robert F. Boyle, and Edgar R. Canavan. Thermal Performance of the XRS Helium Insert, Advances in Cryogenic Engineering (2000) 45507.

4 Koji Kamiya, Brent A. Warner, Michael J. DiPirro, Masahide Murakami. Magnetic Shielding for Sensitive Detectors, To be published in Proceedings of the2000 Innovative Cryogenics Measurements Workshop.

5 E. R. Canavan, J. G. Tuttle, P. J. Shirron, and M. J. DiPirro. Performance of the XRS ADR Heat Switch, Advances in Cryogenic Engineering (2000) 45545.

6 A private communication with R Parmley, Lockheed Palo Research Laboratory, Palo Alto, CA.

\section{FIGURE CAPTIONS}

Figure 1 Illustration of the ADR cycle. Details are provided in the text.

Figure 2 The HAWC/SAFIRE ADR assembly. The superconducting magnet is not shown.

Figure 3 The ADR suspension system assemblies.

Figure 4 The ADR heat switch.

Figure 5 Thermal conductivity data for titanium 15-3-3-3.

Figure 6 Salt pill thermometer spectral noise density during SAFIRE testing. 


\begin{tabular}{|l|l|l|l|}
\hline Instrument & Getter Temp. (K) & Switch Temp. (K) & Conductance (mW/K) \\
\hline HAWC & 14.7 & 1.06 & 14.3 \\
\hline SAFIRE & 16.0 & 1.55 & 31.1 \\
\hline SAFIRE & 16.0 & 4.75 & 41.1 \\
\hline HAWC & 17.4 & 1.90 & 58.6 \\
\hline HAWC & 20.4 & 4.47 & 90.2 \\
\hline
\end{tabular}

Table 1. Closed-state thermal conductances for the HAWC and SAFIRE heat switches. 

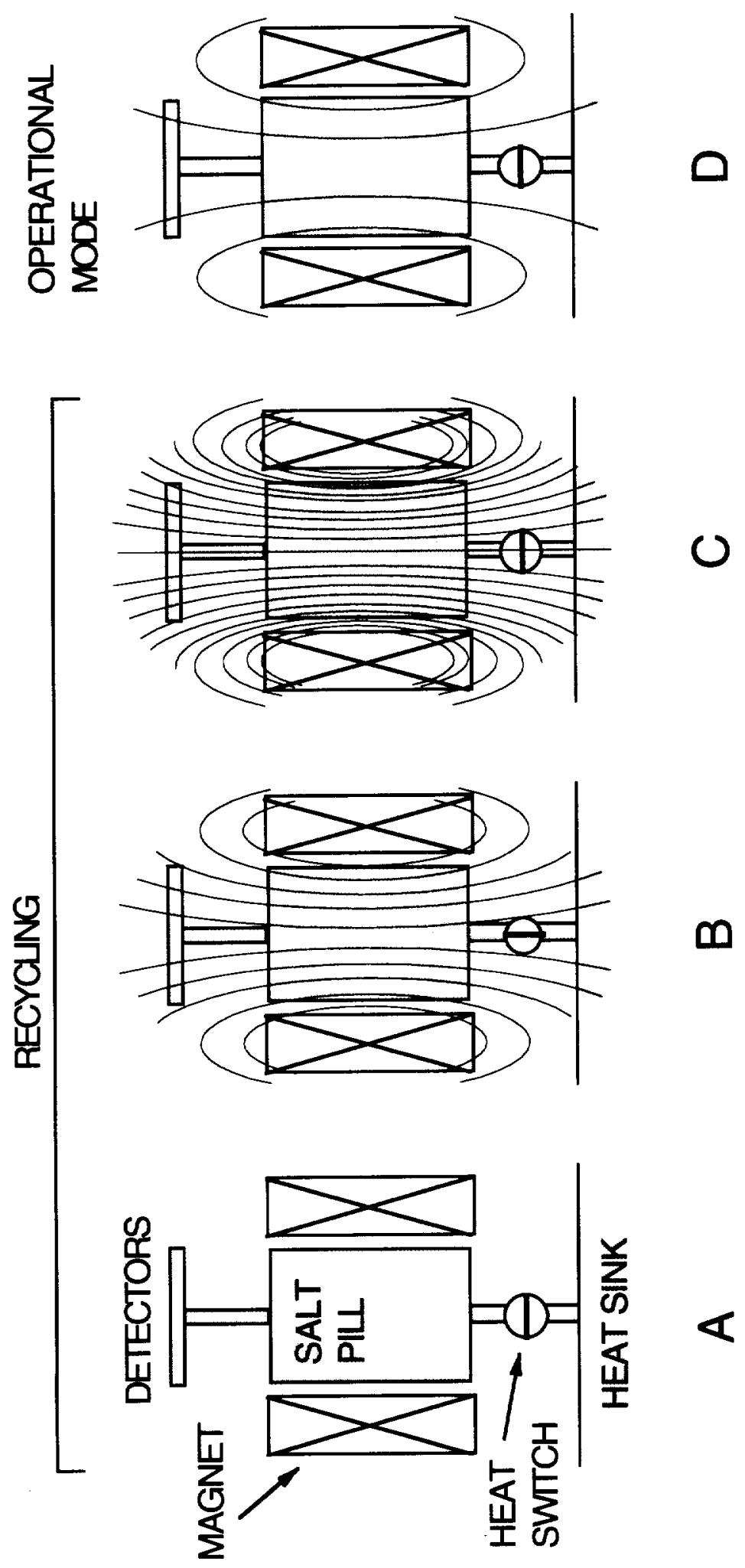


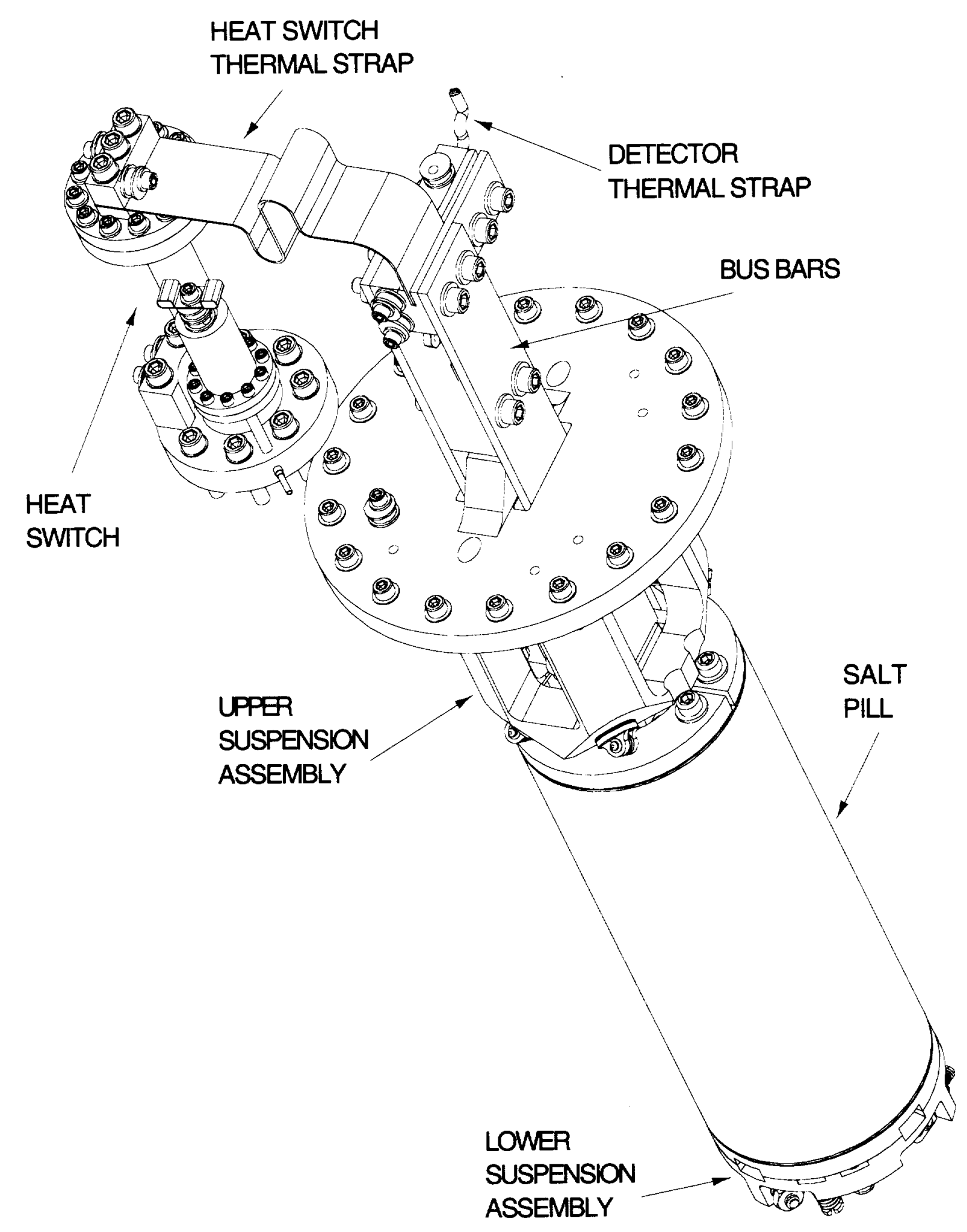

FIGURE 2 


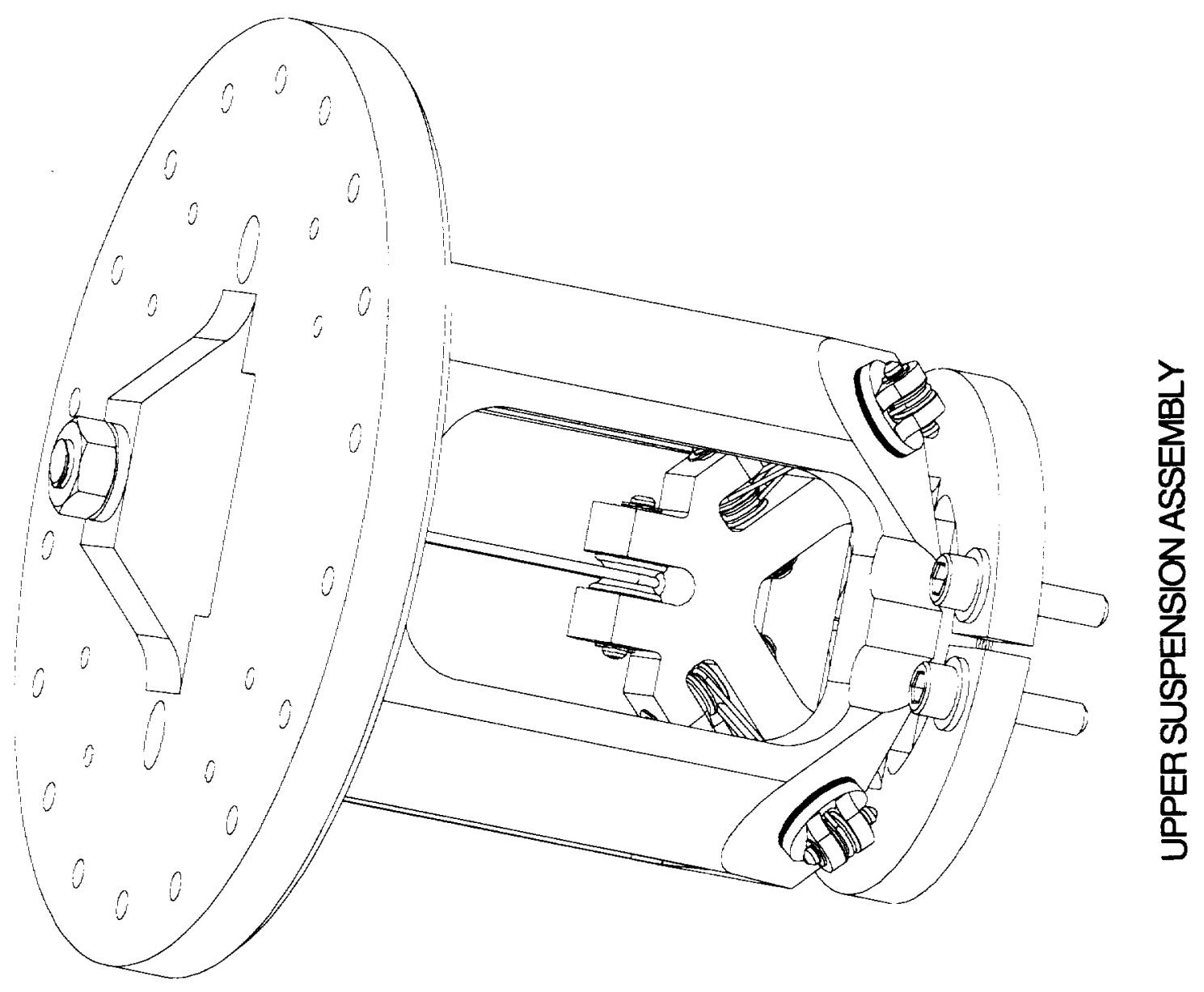

$m$
$w$
3
1
1





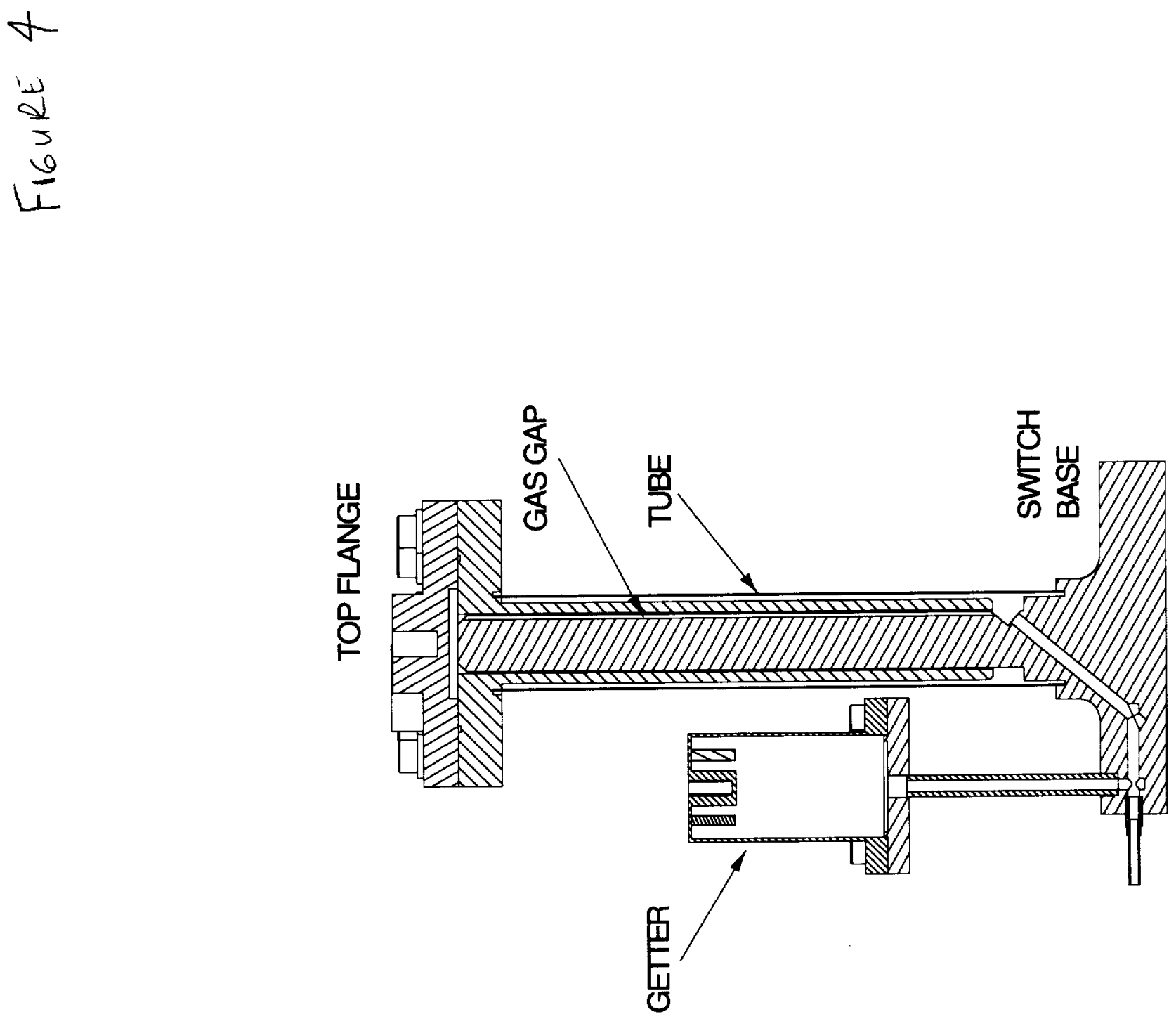

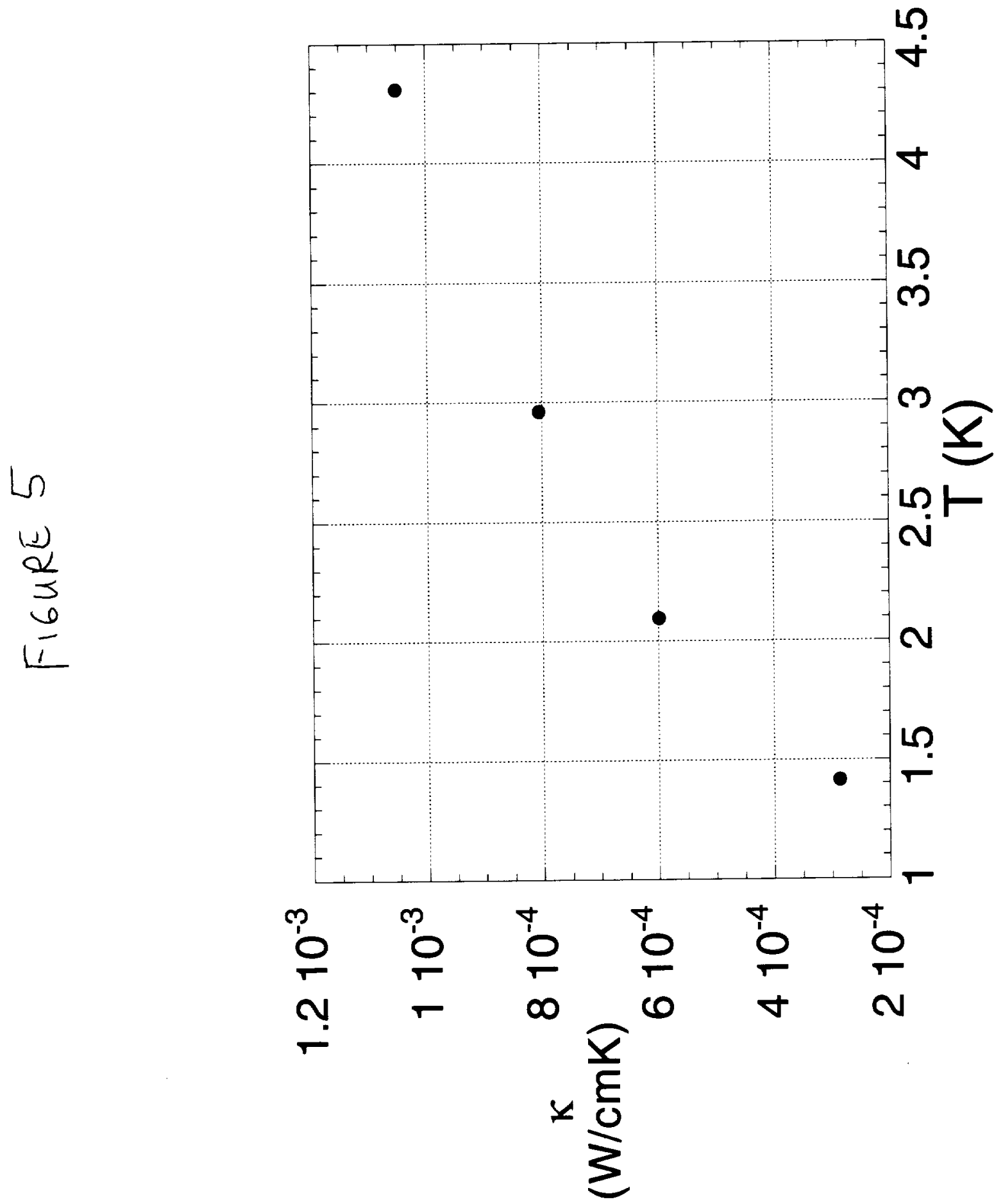


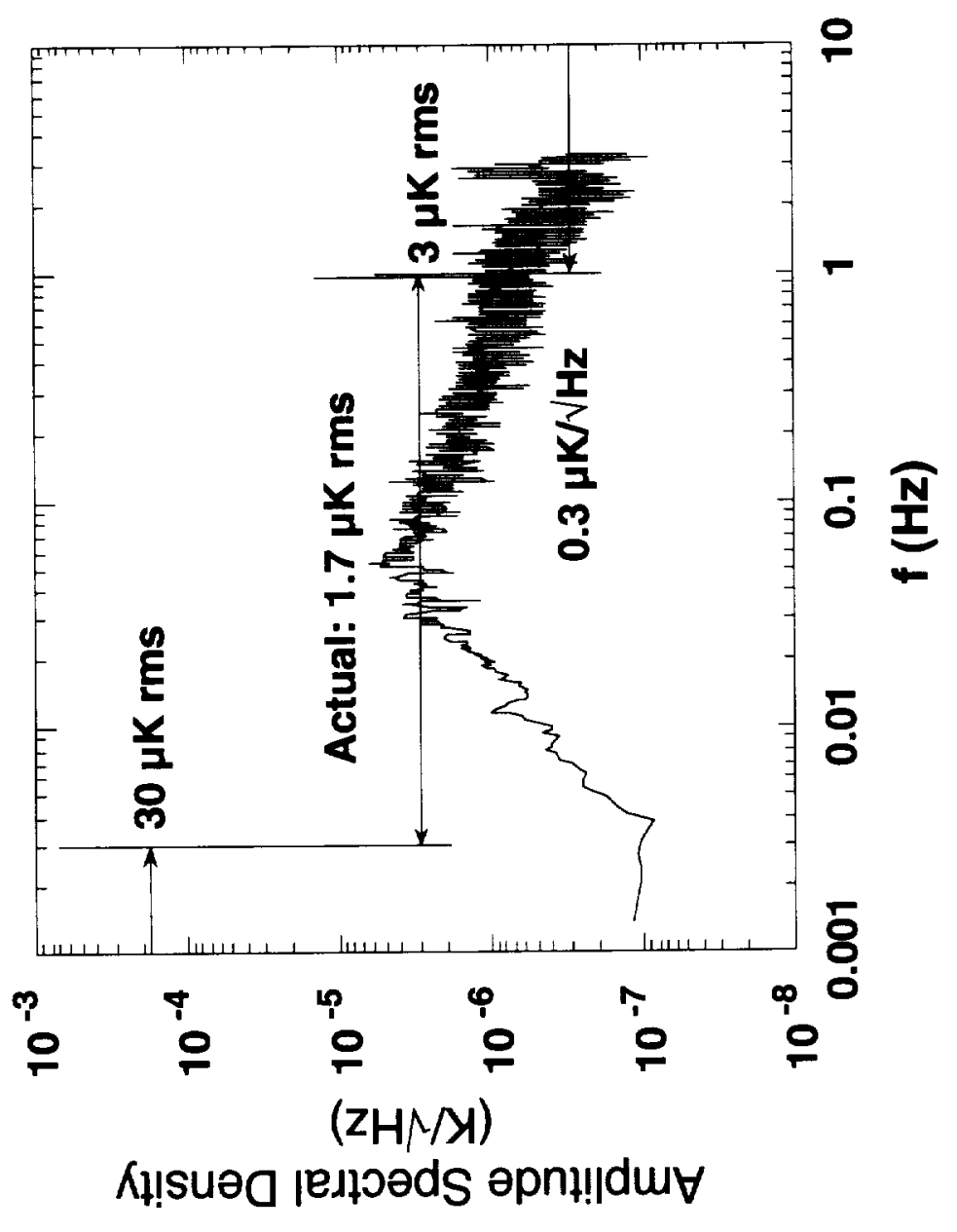

